\title{
Changes in the Total Solar Irradiance and climatic effects
}

\author{
Werner K. Schmutz* \\ Physikalisch-Meteorologisches Observatorium Davos World Radiation Centre, Dorfstrasse 33, 7260 Davos Dorf, Switzerland
}

Received 31 December 2020 / Accepted 16 April 2021

\begin{abstract}
The correlation between the averaged reconstructed March temperature record for Kyoto, Japan, and the reconstructed Total Solar Irradiance (TSI) over 660 years from 1230 to 1890 gives evidence with 98\% probability that the Little Ice Age with four cold periods is forced by variations of TSI. If the correlation is restricted to the period 1650-1890, with two cold periods in the 17th and 19th century and for which two independent reconstructed March temperature records are available, the probability of solar forcing increases to $99.99 \%$. As solar irradiance variations have a global effect there has to be a global climatic solar forcing impact. However, by how much global temperature were lower during these minima and with what amplitude TSI was varying is not accurately known. The two quantities, global temperature and TSI, are linked by the energy equilibrium equation for the Earth system. The derivation of this equation with respect to a variation of the solar irradiance has two terms: A direct forcing term, which can be derived analytically and quantified accurately from the Stefan-Boltzmann law, and a second term, describing indirect influences on the surface temperature. If a small TSI variation should force a large temperature variation, then it has to be the second indirect term that strongly amplifies the effect of the direct forcing. The current knowledge is summarized by three statements:
\end{abstract}

- During the minima periods in the 13th, 15/16th, 17th, and 19th centuries the terrestrial climate was colder by $0.5-1.5^{\circ} \mathrm{C}$;

- Indirect Top-down and Bottom-up mechanisms do not amplify direct forcing by a large amount, i.e. indirect solar forcing is of the same magnitude (or smaller) as direct solar forcing;

- The radiative output of the Sun cannot be lower by more than $2 \mathrm{Wm}^{-2}$ below the measured present-day TSI value during solar cycle minimum.

These three statements contradict each other and it is concluded that at least one is not correct. Which one is a wrong statement is presently not known conclusively. It is argued that it is the third statement and it is speculated that over centennial time scales the Sun might vary its radiance significantly more than observed so far during the last 40 years of space TSI measurements. To produce Maunder minimum type cold climate excursions, a TSI decrease of the order of $10 \mathrm{Wm}^{-2}$ is advocated.

Keywords: Total solar irradiance / TSI / Observations / Radiometry / Space mission / Maunder minimum / Terrestrial climate / Climate variations

\section{Introduction}

Have there been changes in the climate over the last few thousands of years? We all have heard the commonly used terms "Roman climatic optimum", "medieval warm period" or "little ice age". They refer to reportedly unusually warm or cold periods with a duration of the order of several decades to 100 years. Some records show unusual climatic temperatures in certain regions, while others, in particular global averages, hardly show significant climate variability signatures. On the other hand, global climate reconstructions have large uncertainties, and different techniques that are sensitive to different

\footnotetext{
*Corresponding author: werner. schmutz@pmodwrc. ch

This paper belongs to the section "Agora - Scientific Reflections" which allows authors to express their personal views, opinions and experiences on scientific matters related to space weather and space climate.
}

\section{JSWSC}

10 years anniversary issue
Special Issue - 10 years of JSWSC 
seasons do not always agree. On a global scale, historic 100-year-long climate excursions cannot be scientifically identified with significance. Thus, it is not clear if these periods were global events or represent only regional, that is, European climate variations. ${ }^{1}$

However, despite these substantial uncertainties, records attest to a "mild" period in Europe from the years 950-1100 and of "cold winters", at least in northern Europe, in the 16th to 17 th centuries. If we accept that there have been such natural climate variations during the last few thousand years, at least regionally, then the question about the origin of these variations becomes of interest. According to the 100000 -year orbital forcing scenario, the last "abrupt" onset of the warming occurred some 10000 years ago, making the world enter what is called the Holocene (Kawamura et al., 2007; Campisano, 2012). Since this maximum, orbital forcing has slowly been decreasing. As can be noted in temperature records, these effects were highest 6000 to 9000 years before the present time and have decreased since then to a minimum during the most recent pre-industrial time (see Marcott et al., 2013).

Since there is no significant variation in orbital forcing on timescales of 100 or 1000 years, variations in climate must be induced by other phenomena. The influence most commonly proposed by experts are either large volcano eruptions or solar radiance variations, or both. Owens et al. (2017) believe that the timing of climate variations "are not suggestive of causation and should not, in isolation, be used as evidence of significant solar forcing of climate." They put forward "multiple factors, particularly volcanic activity, were crucial for causing the cooler temperatures in the northern hemisphere during the Little Ice Age." However, the influence of even one large eruption lasts only for two or three years. Thus, only a more or less continuous series of eruptions would result in a real volcanic climatic influence. Given that the volcanic frequency deduced from sulfate concentration in ice from Antarctica and Greenland does not support a volcano-only climate forcing (Rypdal \& Nilsen, 2016), it appears that there is a need for additional influence. It is still an open question to identify this additional influence, but the prime candidate is the variation of solar radiance.

A well-known example of a volcanic influence is the eruption of Mount Tambora in Indonesia, on April 10, 1815, which was most probably responsible for the "year without a summer" in the year following the eruption (Arfeuille et al., 2010). But in addition to this identifiable influence in 1816, there was a cool period reported for the northern hemisphere from about 1800 to 1820 that started earlier than the Tambora eruption. Even though another non-localized eruption was reported to have happened in 1809, the cold period extended longer than the effects of both eruptions should have lasted.

Yet, at the same time, there was a period of reduced solar activity, which we can identify from proxies of the solar activity, e.g., reduced sunspot group numbers (Clette et al., 2020). This low solar activity period, lasting from 1790 to 1860 , is called the Dalton minimum. Modeling efforts advocate that the effects of both forcings are needed - volcanic and reduced solar irradiance - to explain the reported cooler climate during the Dalton minimum (e.g., Anet et al., 2013). However, this

\footnotetext{
${ }^{1}$ The introduction follows the thoughts formulated by the author in Chapter 3.2 of Nicollier et al. (2015), a book aimed at laymen interested in space science.
}

example at the beginning of the 19th century has the disadvantage that it covers only a comparatively short period, and so we can therefore barely identify it as a climatic change. On the other hand, this period has the advantage of relatively accurate temperature reconstruction and therefore, we can be quite certain that during the Dalton minimum the temperatures were below average.

To trace the historic solar activity, we rely on proxies, e.g., amongst others, sunspot numbers or the solar modulation potential. Sunspot numbers scale with concentrated strong magnetic fields in the solar photosphere whereas the solar modulation potential is sensitive to open solar magnetic fields. Thus, the two data series are measuring two different solar properties. However, both properties are sensitive to the activity stage of the Sun. For up to 10000 years the solar activity is traced with the solar modulation function, or closely related, the solar modulation potential, and is derived from the cosmogenic radionuclides ${ }^{10} \mathrm{Be}$ and ${ }^{14} \mathrm{C}$ (Abreu et al., 2013). The solar modulation potential is a term introduced to describe the effect of the strength of the solar wind with its embedded open magnetic fields on the average flux of cosmic radiation reaching the Earth (Caballero-Lopez et al., 2019). Measurements of cosmogenic radionuclides span a large time period (the last 10000 years) but have a time resolution of only about 20 years except for the last 100 years. This implies that the sunspot cycles with average periods of 11 years are not resolved, however, the data have enough time resolution to identify grand maxima and grand minima of solar activity.

Proxies for solar activity show that during most of the 17th century, the Sun showed much reduced activity compared to the rest of the 400 years of the telescope era (Usoskin et al., 2014). Except for a possibly normal strength activity cycle at the beginning of the 17 th century - with a maximum around 1613 - sunspots were extremely rare. In particular, in the second half of the century, sunspots were almost completely absent for several decades until about 1710, when the - from our point of view - "normal" sunspot activity started. This normal activity was interrupted briefly, as introduced above, at the beginning of the 19th century during the Dalton minimum. Sunspot numbers then reached a maximum in the middle of the 20th century. The minimum activity period in the second half of the 17 th century is called the Maunder minimum. The Maunder minimum, the Dalton minimum mentioned above, reduced solar activity in the 15th/16th centuries (termed Spörer minimum) and another period of reduced solar activity in the 13th/14th century (called Wolf minimum) coincide with what is called in climate sciences the "Little Ice Age".

While there is widespread agreement that there is a relationship between climatic variations and solar activity variations on the 100 to 1000-year timescale, opinions start to diverge strongly when it comes to identifying the physical reasons and quantifying a potential solar influence. The translation from solar modulation potential to irradiance variations is far from trivial and correspondingly, published reconstructions of solar irradiance differ dramatically. Some scientists argue that observed solar irradiance since 1979, when satellite data became available, has not varied in its basic level. Thus, they exclude the possibility of reduced solar irradiance values and any direct influence of solar irradiance on climate in the past. But there are also other scientists with an alternative view, which has gained wide acceptance. The basic observational fact is that since the 
beginning of the satellite era, solar activity has been stable on a high solar modulation potential level. Thus, with our solar observations over the last 30 years, we have sampled only a rather unusual time period of the Sun. It is possible that solar irradiance values were lower in the past when the solar modulation potential was lower than nowadays. But the community that thinks solar variations are possible splits into different opinions about how large the irradiance variations could have been. Estimates range from irradiance variations, which are insignificant for a potential climate influence, to values with a substantial forcing potential that might explain the climate anomalies during the Holocene (see Fig. 10 and Fig. 8, respectively, of Solanki et al., 2013 and Egorova et al., 2018b). All these reconstructions shown in the cited figures are based on different hypotheses, which are all reasonable, but none of which is proven.

The basic difficulty is that we know about a proxy, which measures the open magnetic field of the Sun but which is not directly related to the physical process that generates irradiance variations. The status of our current knowledge is that the issue around the origin of climate forcing during the Holocene has not yet been conclusively resolved. Similarly, from temperature reconstructions, it is also not known for sure if there were global or regional climate variations within the Holocene larger than fortuitous combinations of natural year-to-year fluctuations. The task of resolving this issue is not only of academic interest but is also important for climate prediction in the future.

\section{Evidence for solar influence on climate}

Aono \& Kazui (2008) and Aono $(2015)^{2}$ were the first to point out that the cold periods of reconstructed temperature records in the 17th century and at the beginning of the 19th century at Kyoto and Edo bay (Tokyo), Japan, agree with reduced sunspot numbers during the Maunder minimum and the Dalton minimum. In Figure 1 averaged terrestrial surface March temperature as deduced from historically recorded dates of cherry blossom are shown. Also included in the figure are annual solar irradiance values as reconstructed by Egorova et al. (2018b). Thus, we are comparing two physical quantities that are connected by the energy equation for the planet Earth (see Sect. 4). Therefore, because of the physical relation, a significant correlation between the two quantities yields irrefutable evidence for the influence of solar forcing on the terrestrial climate.

Since 1650 there are almost uninterrupted records of dates of cherry blossom at both locations, Kyoto and Edo bay. As shown in the Appendix, March temperatures for Kyoto and Edo bay on a 15-year sampling have a standard deviation of $\pm 0.3^{\circ} \mathrm{C}$. Combining the two temperature records results in a relative climate temperature precision of $\pm 0.2^{\circ} \mathrm{C}$, but with a possible systematic trend. The uncertainty is larger during early epochs when flowering dates are more often missing and when only the Kyoto reconstructed temperatures are available and is up to about $\pm 1^{\circ} \mathrm{C}$ at the beginning of the record around the year 1000 .

\footnotetext{
2 The dates of cherry blossom at the two locations and the March temperatures, which were deduced from the blossom dates, have been provided in digital form by Aono (personal communication).
}

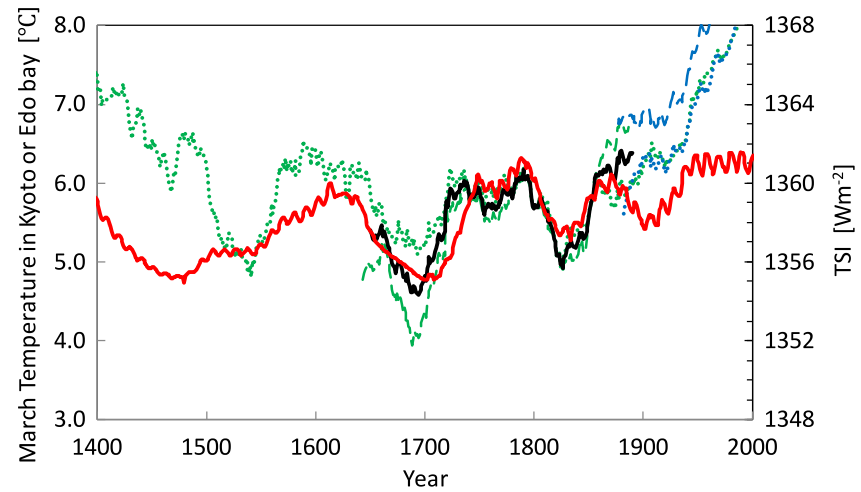

Fig. 1. Reconstructed averaged March temperatures at Kyoto, Japan (green dotted line) adapted from Aono \& Kazui (2008) and Aono \& Saito (2010), where historical records were available since 643 and reconstructed averaged March temperatures at Edo bay (Tokyo), Japan, (green dashed line) starting in 1640 adapted from Aono (2015) using historically recorded dates of cherry blossom ${ }^{2}$. Both time series were smoothed by using a $31-y r$ linear regression. The mean of the temperature curves at the two locations is illustrated by the thick black line. The blue lines starting in 1880 show thermometer measured temperatures in Kyoto (dotted line) and Edo bay (dashed line) averaged in the same way. Also shown is the 21-year reconstructed TSI as published by Egorova et al., 2018b (red line, scale on the right axis).

After the year 1650, not many years have a missing blossom record and the shown temperature variations are significant at both locations. The averaged March temperature curves of these two locations are illustrated by the dotted and dashed green curves respectively. Kyoto and Edo bay are separated by $500 \mathrm{~km}$ and there is a systematic trend and offset between the Kyoto and Edo bay curves, indicating a systematic bias between the two locations, which is also found in measured temperatures at those locations in the 20th century (see the Appendix). However, overall the reconstructed temperature trends at the two locations are similar in the common interval 1650-1890 and the correlation of the two climatic temperature trends is strong and highly significant $(R \approx 0.8, P<0.02 \%)$. Thus, the mean of the two locations, which is shown by the thick black line, reflects climatic March temperature variations of the Japanese region from Kyoto to Edo bay. The reconstructed TSI variations as published by Egorova et al. (2018b) reveal the same variation pattern as the mean of the two locations, reproducing even the same relative amplitudes for the Maunder and Dalton minima. The correlation between the mean of the Kyoto and Edo bay climatic temperature variations and the reconstructed TSI is as strong as the correlation between the temperatures records of the two locations and the probability that this correlation is by chance, i.e. the probability of the zero hypothesis, is less than $P<0.01 \%$. Thus, the probability for an influence of solar forcing on the mid-southern region in Japan for the epoch 1650 1890 is larger than $99.99 \%$ and testifying an influence of solar forcing on terrestrial climate beyond a reasonable doubt.

For the years earlier than 1650 a correlation between averaged March temperature and TSI relies on temperature reconstruction for Kyoto only. When going back in time there are more and more annual records missing and therefore, the reliability of the climate temperature record is declining. In the 
Appendix, Table A.1 gives correlation coefficients for various epoch ranges. The correlation of larger epoch intervals to solar forcing becomes less and less significant unless one accounts for a biased trend of the temperature record. Given that the uncertainties of both, the temperature reconstruction and TSI reconstruction become higher before 1600 , this does not necessarily imply that the correlation of the reconstructed temperature with TSI is fortuitous. As the influence of a biased trend is to be expected because the differences between Kyoto and Edo bay are showing this, a multiple linear regression analysis is justified. If a biased trend is included then the regression analysis of the reconstructed temperature record for Kyoto yields a significant influence of solar forcing $(R=0.54, P=1.6 \%)$, over more than 600 years from 1230 until 1890.

In the 20th century, there are thermometer-measured March temperatures available at the two locations. Only the Kyoto curves show an initial tendency to follow the TSI curve to cooler temperatures and then both measured temperature records are steeply rising. Since the early 20th century both locations are influenced by urban warming (Omoto \& Aono, 1991) and after mid 20th century by global warming. It is therefore not surprising that there is no clear relation between solar activity and cherry blossom dates in these two cities during the last century.

The amplitude of the temperature variations is remarkably high. The "normal" average preindustrial March temperature at Kyoto is about $6^{\circ} \mathrm{C}\left(1720-1800: 6.0 \pm 0.1^{\circ} \mathrm{C}\right.$; today the average March temperature is about $8^{\circ} \mathrm{C}$ ) and we see in Figure 1 the Kyoto temperature during the Maunder minimum decreased by $1{ }^{\circ} \mathrm{C}$. The Edo March temperature curve decreases to about $4{ }^{\circ} \mathrm{C}$, indicating average March temperatures during the Maunder minimum to be colder by $2{ }^{\circ} \mathrm{C}$. The mean temperature decrease during the Maunder minimum is about $1.5^{\circ} \mathrm{C}$ but the uncertainty of this amplitude is of the order of a factor of two as indicated by the difference between the two cities as well as the bias trends found in the regression analyses presented in the Appendix.

The coefficient $c_{\text {TSI }}$ given in the Appendix, connecting the reconstructed irradiance with the reconstructed temperature, is in the principle of high interest because it gives information by how much direct solar forcing is amplified by indirect forcing. However, the uncertainty of this coefficient is given by a factor of two for the temperature amplitude and another factor of two for the amplitude of the irradiance reconstruction (Shapiro et al., 2011). Taken together the uncertainty prevents a conclusion about solar forcing amplification.

It would be a bold extrapolation to conclude from the amplitude of a regionally reconstructed March temperature that the global climate was variable with this amplitude. However, the argumentation can be reversed: Given the solar influence over the 1230-1890 time range is demonstrated, everywhere on Earth this influence must have had an impact. Also given that the solar forcing is not very variable on annual to decadal time scales but that it takes a few decades to change by a large amount, it does not matter in what season a climate record is evaluated. The task at hand is to identify a location, which is representative of a global temperature change. It is argued in the following that the mid-Japanese region is representative.

As the temperatures in middle-southern Japan in general, and in particular at Edo bay are influenced by the average Pacific sea temperature, it is plausible that this Japanese temperature reconstruction is not far away from the amplitudes of a global climate temperature record. Based on climate simulations with the model SOCOLv4, which is based on the MPI-Met Earth System model (Sukhodolov et al., 2021), run from 1980 to 2099 , a very close agreement between the temperature variations in the Japanese Kyoto-Tokyo region and northern hemisphere as well as global temperatures means is verified (Egorova et al., 2018a, 2019; Rozanov, personal communication). For the time of the Dalton minimum in the early 19th century, there are measured temperatures in England that confirm cold temperature excursions for 10-year-mean temperature values of the same magnitude as given above for Japan (see Fig. 1 of Lübken \& Nicoll, 2016), although in England in the 19th century there have been additional periods that had a similar temperature decline. Overall, the conclusion is that decadal-averaged global temperature reductions during the Maunder and Dalton, and likely also during the Wolf and Spörer solar minima have a magnitude of the order of a degree and not fractions of a degree.

\section{Measurements of the total solar irradiance}

Because TSI is the energy input to Earth, an accurate measurement record of the spectrally integrated TSI arriving at the top of the atmosphere is very important for the assessment of the Earth's climate system. High precision measurements of TSI in space started in 1979 and since then, an (almost) uninterrupted series of measurements has been maintained. Unfortunately, all space instruments suffer from degradation and this is particularly true for those instruments, which observe the full spectrum of the Sun including its UV radiation, which is harmful to detector systems (BenMoussa et al., 2013). Since about the mid-1980s measures are in place for assessing the sensitivity change of the instruments during their exposure to the Sun's light. Nevertheless, it is not straightforward to evaluate by how much the measured irradiance records have to be corrected, which leads to different opinions on how data are to be combined (Willson \& Mordvinov, 2003; Dewitte et al., 2004; Fröhlich, 2013).

Arguably the best-estimate composite of TSI from the beginning of the space era in 1979 until 2003 is from Fröhlich (2006), commonly called PMOD-composite. This opinion is based on the author's intimate knowledge of all TSI experiments flown in space. After SORCE/TIM came into operation (Kopp et al., 2005), a less personal approach is better suited to merge TSI records from different experiments, which is done, e.g., by the method of Dudok de Wit et al. (2017), termed here TDdW-composite. The published time series of the latter publication extends until 2015. Updates of merged TSI records are in preparation $^{3}$ but not yet published. Therefore, the merged TSI record shown in Figure 2 uses the mean of the values of version 18 of SORCE/TIM ${ }^{3}$ and the PMOD-composite until mid-2017. In this time interval, the PMOD-composite is identical to the measurements obtained by SOHO/VIRGO. From 2017 the SORCE/TIM measurements are used until the end of 2019. The data in 2020 are the measurements of TSIS/TIM. ${ }^{4}$ The

\footnotetext{
${ }^{3}$ Greg Kopps TSI Page. Webpage https://spot.colorado.edu/ $\sim$ koppg/TSI/, access November 2020.

${ }^{4}$ TSIS-1. Webpage https://lasp.colorado.edu/home/tsis/data/tsi-data access November 2020
} 


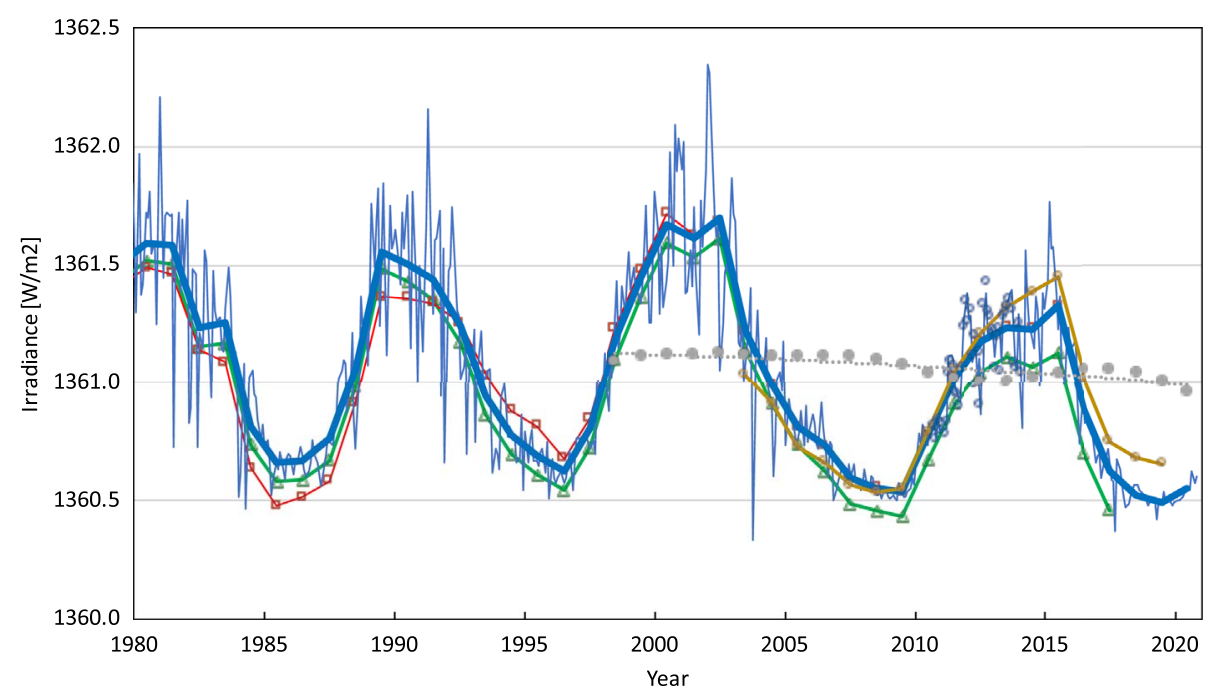

Fig. 2. Time series of measured TSI values. The light blue thin line shows monthly means and yearly averages are denoted by the thick blue curve. This TSI-composite is a version by the author (see text for the merging procedure). Also shown are other TSI-composites: The PMOD TSI-composite (Fröhlich, 2006, thin green line with open green triangles), the composite of Dudok de Wit et al. (2017, red line, open red squares), and the measurements of SORCE/TIM (Kopp et al., 2005, thin gold line, open gold circles). The filled grey circles illustrate a mean over 21-years with the entry plotted at the last year of the interval.

transition from one data set to the other is obtained from a linear fit to the difference of a one-year overlap period before the transition and then using the offset of the fit at the transition date to shift the more recent data set to continue the composite.

The absolute calibration is adapted from the TDdWcomposite, which is based on the absolute values of SORCE/TIM (Kopp \& Lean, 2011) and PREMOS/PICARD (Schmutz et al., 2013) at the first light of PREMOS in July 2010. In Figure 2 PREMOS monthly mean values are marked by open blue circles from 2010 until 2014. On the scale of this figure the PREMOS values are identical with the TDdW-composite. The yearly averages of the SORCE/TIM measurements are illustrated by open gold circles connected by a thin line in gold, which is in 2010 in excellent agreement with the PREMOS values. Also shown in Figure 2 is the PMOD-composite (version 42651709 Fröhlich, 2006, green open triangles, thin green line) in its full length and on its published "VIRGO" TSI-scale until the end of this data set in 2017. The full published length of the TDdW-composite record from its beginning in 1979 (here shown from 1980) until 2015 is illustrated by the red curve with open red squares.

The differences between the different merged TSI time series illustrate the difficulties faced when stitching together different experiments. The time series shown in blue, put together by the author, is not more accurate than any other published record: Any differences are within the uncertainties of these records. The PMOD-composite suggests that there was a decline of $0.1 \mathrm{Wm}^{-2}$ between the TSI-minima in 1985 and 2017 (the last data point date shown by the green triangle), whereas the blue TSI-composite line yields a decline of $0.2 \mathrm{Wm}^{-2}$ between 1985 and 2019. The measurements of SORCE/TIM suggest an increase from the minimum in 2009 to 2019. Neither trend is significant as the uncertainties of the records, about $0.1 \mathrm{Wm}^{-2} / 10 \mathrm{yr}$, have larger magnitudes. The difference of the curves in 2019 can be traced back to the fact that the
SOHO/VIRGO measurements and the SORCE/TIM record have different long-term trends during solar cycle 24 , which accumulates to the resulting difference. It is astonishing by how much the trends of the two experiments differ as both experiments are based on four sensors that should ensure long-term stability of the TSI record. The obvious conclusion is that currently, it is not possible to be confident of any multi-decadal trend in TSI.

\section{The magnitude of a direct influence of Total Solar Irradiance}

The idea that the Sun influences the Earth directly by a varying energy deposition on Earth has already been formulated in the 19th century (see references given by Eddy et al., 1982). In the first half of the 20th century, the director of the Smithsonian Institution was instrumental in advancing ground-based monitoring of the solar irradiance and he became convinced that changes of the solar radiance influence the terrestrial weather (Abbot, 1950). In the second part of the 20th century, the idea of a solar influence on the weather was dismissed by Eddy et al. (1982) on grounds of the small magnitude of the measured solar irradiance variations, which were quantified on the $0.1-0.3 \%$ level in Eddy's paper for variations on a weekly time scale due to large sunspot groups.

To quantify the direct solar influence we use the textbook formula for the terrestrial energy balance

$$
\pi \mathcal{R}_{\oplus}^{2} \mathcal{S}_{\odot}\left(1-\mathcal{A}_{\oplus}-\alpha\right)=4 \pi R_{\oplus}^{2} \sigma T_{\oplus}^{4}\left(1-\mathcal{G}_{\text {eff }}\right),
$$

where the left-hand side is the solar energy that reaches the terrestrial ground level and the right-hand side gives the outgoing radiation energy. $T_{\oplus}$ is the average terrestrial surface temperature, $R_{\oplus}$ Earth's radius, and $\mathcal{S}_{\odot}$ is the total irradiance received on Earth at the average distance of $1 \mathrm{AU}$ from the 
Sun. $\mathcal{A}_{\oplus}$ denotes the terrestrial albedo, $\alpha$ the absorption of radiation when passing down through the atmosphere, and $\mathcal{G}_{\text {eff }}$ stands for an effective Greenhouse effect that includes everything of Earth's highly complex climate system; i.e. not only back radiation, latent, and sensible heat but also absorptive, dissipative, and distributional effects that are responsible for the negative entropy flux into the Earth's climate system (Aschwanden et al., 2018). The average solar irradiance over the past three decades is $\mathcal{S}_{\odot}=1361 \mathrm{~W} / \mathrm{m}^{-2}$ (Prša et al., 2016). The albedo and atmospheric absorption are given as $\mathcal{A}=29.4 \%$ and $\alpha=23.5 \%$ in the global energy balance diagrams (e.g., Wild et al., 2015). In order to get the current average terrestrial surface temperature, say $288 \mathrm{~K}$, the effective Greenhouse effect is $\mathcal{G}_{\text {eff }}=59 \%$.

The climate sensitivity to a varying solar irradiance is given by

$$
\begin{aligned}
\frac{\partial T_{\oplus}}{\partial \mathcal{S}_{\odot}}= & \frac{\partial}{\partial \mathcal{S}_{\odot}}\left(\sqrt[4]{\frac{\left(1-\mathcal{A}_{\oplus}-\alpha\right)}{4 \sigma\left(1-\mathcal{G}_{\mathrm{eff}}\right)}}\right) \cdot \sqrt[4]{\mathcal{S}_{\odot}} \\
& +\sqrt[4]{\frac{\left(1-\mathcal{A}_{\oplus}-\alpha\right)}{4 \sigma\left(1-\mathcal{G}_{\text {eff }}\right)}} \cdot \frac{\partial \sqrt[4]{\mathcal{S}_{\odot}}}{\partial \mathcal{S}_{\odot}}
\end{aligned}
$$

where the first term is indirect solar forcing and the second term is direct solar forcing. Assuming that the parameters $\mathcal{A}_{\oplus}, \alpha$, and $\mathcal{G}_{\text {eff }}$ of the equation for the global energy balance are not affected by a variation of the solar irradiance, i.e. $\partial \mathcal{A}_{\oplus} / \partial \mathcal{S}_{\odot}=\partial \alpha / \partial \mathcal{S}_{\odot}=\partial \mathcal{G}_{\text {eff }} / \partial \mathcal{S}_{\odot}=0$, we obtain

$$
\frac{\partial T_{\oplus}}{T_{\oplus}}=\frac{1}{4} \frac{\partial \mathcal{S}_{\odot}}{\mathcal{S}_{\odot}},
$$

which yields

$$
\partial T_{\oplus}=f \partial \mathcal{S}_{\odot},
$$

with $f=T_{\oplus} /\left(4 \mathcal{S}_{\odot}\right)=0.053{ }^{\circ} \mathrm{C} /\left(\mathrm{W} / \mathrm{m}^{2}\right)$, the sensitivity parameter to variations of the solar irradiance for the present day values of $T_{\oplus}$ and $\mathcal{S}_{\odot}$ as given above. ${ }^{5}$

The largest observed radiance variations by the Sun result from the passage of very large sunspot groups. In the space era, the largest observed decrease in TSI was the end of October 2003 , when there was a TSI reduction by $0.34 \%$, which corresponds to $4.6 \mathrm{~W} / \mathrm{m}^{2}$. According to equation (4), this forces a decrease of $1 / 4{ }^{\circ} \mathrm{C}$ of the terrestrial temperature. This forcing lasts only a few days and because the daily weather variability is much larger, this forcing is probably not detectable in global temperature records.

The largest measured irradiance variations result from the eccentric orbit of Earth around the Sun. The present-day eccentricity is $e=0.0167$ (Urban \& Seidelmann, 2012), which yields a ratio of aphelion to perihelion distances of $(1+e) /(1-e)=$ 1.034 that makes the solar irradiance measured at Earth vary from $1316 \mathrm{~W} / \mathrm{m}^{2}$ in July to $1407 \mathrm{~W} / \mathrm{m}^{2}$ in January. This is a peak-to-peak amplitude of about $90 \mathrm{~W} / \mathrm{m}^{2}$, which would make the global temperatures vary by almost $5{ }^{\circ} \mathrm{C}$ over a year because of the changing distance to the Sun. This is not observed due to

\footnotetext{
5 Note that in climate sciences the incoming solar energy is usually related to the total Earths surface and thus, the solar irradiance is divided by a factor of four, $\hat{\mathcal{S}}_{\odot}=\mathcal{S}_{\odot} / 4=340 \mathrm{~W} / \mathrm{m}^{2}$, which yields a climate sensitivity $\partial T_{\oplus}=f \partial \hat{\mathcal{S}}_{\odot}$, with a factor of four larger numeric value $\hat{f}=0.21^{\circ} \mathrm{C} /\left(\mathrm{W} / \mathrm{m}^{2}\right)$.
}

the influence of the larger land area on the northern hemisphere and the damping effect of the heat absorption by the oceans.

The integrated radiation energy received over one year does not depend on eccentricity to first order, but only to second order: there is only a little more energy for a larger eccentricity. Thus, orbital forcing is not a matter of the total energy, but of the season in which it is received. As is well known, this forcing has a crucial impact on the glacial and interglacial cycles. The empirically established relationship between orbital influence and the onset of warm periods shows that the insolation received during the northern summer matters. The climate gets stronger forcing towards a warmer climate when perihelion occurs in northern summer (Schmutz \& Haberreiter, 2016).

In Section 3 we have seen that the yearly averaged TSI increases by $0.5-1 \mathrm{~W} / \mathrm{m}^{2}$ from solar cycle minimum to maximum. This would yield an increase of the terrestrial global temperature on $\Delta T_{\oplus}=0.02-0.05{ }^{\circ} \mathrm{C}$, depending on the TSI amplitude of a particular solar cycle. The important question is whether the duration of the maximum is long enough to force a changed climate equilibrium to be established. This question is addressed below in the next section.

On solar cycle averages, the observed TSI record is stable over decades within a range of $\pm 0.1 \mathrm{~W} / \mathrm{m}^{2}$, which implies that a global temperature trend due to a long-term trend of TSI over the last 30 years is $\Delta T_{\oplus}<0.01{ }^{\circ} \mathrm{C}$. This invalidates any claim that global warming could be forced by the Sun.

Contrasting to all the small solar influences on the global temperature discussed above, we have seen in Section 2 indications that the climate in the 17 th century may have been colder by as much as $1^{\circ} \mathrm{C}$, which requires according to equation (4) if this temperature reduction is forced only by the direct forcing term, a decrease of TSI by $20 \mathrm{~W} / \mathrm{m}^{2}$.

\section{Indirect solar forcing}

The assessment made so far brings a contradictory situation in that climate indicators in the 17th and 19th centuries seem to point to periods that were cooler than average and evidence points to a solar influence forcing these cold periods, which is contrasted by the observational fact that for the last 40 years no influence of the Sun on climate has been identified. Yearly averages of TSI are well correlated with the yearly averages of the sunspot number $(R \approx 0.94)$. Therefore, as in the Maunder minimum in the 17th-century sunspots have been absent (Eddy, 1976), i.e. the sunspot number was essentially zero for decades, we may conclude that TSI assumes a more or less constant value at the observed TSI values corresponding to $\mathrm{SSN}=0$ (see Sect. 3); i.e. TSI $\mathrm{SSN}=0 \approx 1360.5 \mathrm{~W} / \mathrm{m}^{2}$ over decades during the Maunder minimum. Compared to the present day 21-year TSI average, $\mathrm{TSI}_{\langle 21 \mathrm{yr}\rangle} \approx 1361.0 \mathrm{~W} / \mathrm{m}^{2}$, this is $0.5 \mathrm{~W} / \mathrm{m}^{2}$ lower and according to Section 4 , the direct forcing of this decreased TSI yields a global temperature reduction of $\approx 0.03{ }^{\circ} \mathrm{C}$. Therefore, in order to force $\approx 1{ }^{\circ} \mathrm{C}$ cooler temperatures during the Maunder minimum the contribution from the term for indirect solar forcing, the first term in equation (2), had to be 30-times larger than the direct forcing term, i.e. indirect forcing by non-zero partial derivatives of the parameters $\mathcal{A}_{\oplus}, \alpha$, and $\mathcal{G}_{\text {eff }}$, had to dominate the proposed climate change.

The climate literature refers to two ways of amplifying direct TSI-forcing, termed "Bottom-up" and "Top-down" mechanisms 
(see Matthes et al., 2016; Maycock \& Misios, 2016, and references therein). As the terms suggest, the first one involves a reaction of the sea temperature, which is inherently a slow process that take years to develop, whereas the second mechanism takes place in higher atmospheric layers with all processes being fast. We note that monthly averages of the global temperature do not show a signature of the annual TSI amplitude of $90 \mathrm{~W} / \mathrm{m}^{2}$ due to the eccentric Earth orbit. The average monthly global temperature curve of the last 160 years has a $0.1{ }^{\circ} \mathrm{C}$ minimum to maximum amplitude with the maximum occurring in August and not at the beginning of the year when the irradiance is largest. On the one hand, this signifies that the Top-down mechanism, which is fast enough to respond within a month time scale, is not amplifying direct solar forcing by a large factor because otherwise monthly averages of global temperature would show a much larger temperature amplitude. On the other hand, the missing impact within a monthly time scale implies that the Bottom-up mechanism needs more time to develop because from energy considerations a $90 \mathrm{~W} / \mathrm{m}^{2}$ forcing cannot be without a reaction over longer time scales.

Yearly averages of the global temperature do not show a clear signature of the forcing by the $0.5-1 \mathrm{~W} / \mathrm{m}^{2}$ variations from solar cycle minimum to maximum. As was for the annual cycle, this observational fact also excludes a significant amplification by the Top-down mechanism. However, given that the maximum of a solar cycle lasts in the order of three years, it limits the Bottom-up mechanism as well. If there would be an, e.g., three-fold or larger amplification, it would lead to an unambiguously observable global temperature oscillation correlated with the 11-year solar cycles. A Fourier transformation of annual global near-surface temperatures (HadCRUT5 Morice et al., 2020), detrended for global warming with a third-degree polynomial, shows a peak with a period of 9.1 years. If this peak is related to solar cycle forcing is not clear. Evidence for a relation to solar cycle forcing is that this periodicity has an amplitude of $0.05^{\circ} \mathrm{C}$, which is anticipated from forcing by $1 \mathrm{~W} / \mathrm{m}^{2}$ direct forcing. There is also power in the periods of $1 / 2$ solar cycle and twice the solar cycle, which might be responsible for the difference of the $9.1 \mathrm{yrs}$ peak to an expected $11 \mathrm{yr}$ periodicity. The strongest peak with a $0.1{ }^{\circ} \mathrm{C}$ amplitude is at 64 yrs resulting mainly from the temperature hump around the year 1945. As this 1945 hump is very obvious to the eye this demonstrates that even moderate amplification of the direct forcing would be detectable.

Indications for a weak signature of solar cycle forcing have already been found in the re-analyses of reconstructed sea surface temperatures (van Loon et al., 2007) but as above, for the global averages, it is not clear if the detected patterns are significant evidence for solar cycle forcing. In any case, solar cycle signatures are not strongly amplified by large factors and in no way by a 10 -fold amplification.

Over the past two decades, climate models have been improved to model the impact of solar forcing. Emphasis has been made to include the impact of the variations of the solar spectrum, in particular the UV variations, to model realistically the Top-Down mechanism, and the inclusion of ocean modeling has allowed investigating the Bottom-up mechanism (Sukhodolov et al., 2014; Matthes et al., 2016). Climate simulations of the Dalton minimum at the beginning of the 19th century yielded the result that Top-down solar forcing only has an insignificant impact on the climate during the Dalton Minimum but that Bottom-up forcing has led to a decrease of up to $2 \%$ of the ocean heat content for forcing by about $-10 \mathrm{~W} / \mathrm{m}^{2}$, due to the reduced atmospheric transmission of volcanic aerosol emissions. This TSI decrease led to a global cooling of about $0.8^{\circ} \mathrm{C}$ (Anet et al., 2014). A similar result is found by Egorova et al. (2018b) by investigating the first half of the 20th century. These results indicate that according to model calculations the two terms in equation (2) have about the same order of magnitude, i.e. the direct solar forcing is amplified by at most a factor of two but not more than that.

Reconstruction of TSI based on so-called physics-based models (see, e.g., Kapyla et al., 2016, and references therein) is very successful in explaining the observed TSI variations as a result of a varying magnetic activity up to the very details of the TSI modulation that can be negative for sunspots as well as positive for flares (Shapiro et al., 2017). In principle, these reconstructions have no lower limit for the TSI corresponding to minimal magnetic activity. However, in practice, because based on today's observational resolution, it is found that the magnetic content of the quiet Sun is found to be not much larger than about the amplitude to the maximum, the lowest limit to the dimmest state of the Sun has been derived to be about $2 \mathrm{~W} / \mathrm{m}^{2}$ below the 2019 minimum (Yeo et al., 2020). Thus, the reduction of a 31-year average TSI during the Maunder minimum relative to today's TSI would be about $2.5 \mathrm{~W} / \mathrm{m}^{2}$. This amplitude is five times larger than what has been discussed above but there is still a need for amplifying direct solar forcing by a factor of eight. Given the observational evidence that amplification of direct solar forcing is not more than a factor of two, this is in direct contradiction to the observation that Maunder minimum global temperature reduction was of the order of $1{ }^{\circ} \mathrm{C}$. The question remains open whether there would be a larger amplification for forcing that has time scales of decades rather than years, as for volcanic forcing, or a few years for solar cycle forcing.

\section{Conclusions}

Evidence for solar influence on climate is reported from a multitude of different sources (see e.g., contributions in Dudok de Wit et al., 2016). A particular intriguing correlation between a climate indicator, the date of cherry blossom at two locations in Japan, and solar activity has been presented in Section 2. In that example, the Maunder minimum, as well as the Dalton minimum of solar activity, is related with $99.99 \%$ probability to climatic cold periods and with $98 \%$ probability also to the Wolf and Spörer solar activity minima. The next important question is how large this influence is in terms of a global temperature anomaly. In Section 2 it was argued that the amplitude of the temperature anomaly is about one degree rather than fractions of a degree. Such a large anomaly is not surprising in that it needs a large signal to be detected within the variability of weather. But then again, a large climate signal needs a strong forcing that has not yet been identified.

From the combined TSI measurements since 1979 we learn that the yearly averaged TSI time series have amplitudes from minimum to maximum of the solar activity cycles 22 and 23 of about $1 \mathrm{Wm}^{-2}$ and about half this value in the last cycle 24. A 21-year average TSI-trend is stable within $\pm 0.05 \mathrm{Wm}^{-2}$ exhibiting only a weak decline between 1990 and 2010 of 
$0.1 \mathrm{Wm}^{-2}$, due to the reduced solar activity with a reduced TSI maximum during the last solar cycle. This reduction in the 21-yr average is real because the reduction in TSI amplitude from minimum to maximum in cycle 24 relative to previous cycles is larger than the measurement uncertainties. However, the reduction in the 21-yr averaged TSI is so tiny that there is no consequence for the terrestrial climate. No additional trend can be seen in the long-term of minima TSI values that are larger than the time series' uncertainty of $0.2 \mathrm{Wm}^{-2}$. A solar constant value can be set at $1361 \mathrm{~W} / \mathrm{m}^{2}$ as has been adopted by the IAU 2015 Resolution B3 Prša et al. (2016).

From the energy conservation equation of the Earth system, the effect of solar variability on climate has two terms: A direct effect that relates a variation in TSI to the global temperature and a term, which summarizes many potentially non-linear influences on Earth's climate. The climate community talks about "Top-down" and "Bottom-up" mechanisms and in the context of the solar influence, these mechanisms amplify the impact of the direct solar forcing. An important characteristic of amplification is whether it is a fast reaction that should show up immediately or if it needs time to build up. All processes in the atmosphere are fast in the sense that new status is established within minutes for radiative processes and within hours to days for atmospheric waves and wind patterns. In fact, in higher atmospheric layers the Top-down mechanism is easily detected. If it existed, a large amplification of direct forcing by Top-down mechanism on near-surface temperatures would be observable, in particular amplifying the huge annual variation due to the eccentric Earth orbit about the Sun. From the missing impact of solar irradiance variations with time scales shorter than months, it can be concluded that the Top-down mechanism does not have a significant impact on the global surface temperature. This is confirmed by climate model simulations that only produce negligible Top-down forcing despite the efforts of the past decades to build climate models that treat the Top-down mechanism.

For the Bottom-up effect, the direct forcing has to build up with time to make an integrated difference in energy compared to the large energy content of the ocean. If the forcing is large enough, e.g., for large volcanic events that yield a global reduction in atmospheric transmission due to their aerosol emissions, there are reported observable effects on climate. Thus, we know that within one or two years a TSI forcing is detectable. From the at-best marginally detected solar cycle influence on near-surface temperatures we conclude that a large amplification of the direct forcing does not exist; neither for Top-down nor for Bottom-up mechanisms.

As presented in Section 5, physics-based models of TSI variations limit a maximal TSI decrease to $\Delta S \leq 2.5 \mathrm{~W} / \mathrm{m}^{2}$ compared to today's mean TSI value.

From the findings above three statements can be formulated:

- Forced by reduced solar activity during the Maunder minimum period in the 17 th century the terrestrial climate was colder by about one degree;

- Indirect Top-down and Bottom-up mechanisms do not amplify direct forcing by a large amount, i.e. indirect solar forcing is of the same magnitude (or smaller) as direct solar forcing;
- The radiative output of the Sun cannot be lower by more than $2 \mathrm{Wm}^{-2}$ below the measured present-day TSI value during solar cycle minimum.

A comparison of these statements yields that the second and third statements, taken together, contradict the first. This contradiction is only resolved if it could be shown that one of the three statements is incorrect, which one cannot be decided conclusively.

There is one science community, which has made a different approach to derive the potential amplitude of the solar radiance to vary: Shapiro et al. (2011) and Egorova et al. (2018b) have argued that the radiance distributions on the quiet Sun regions reflect the amount of magnetic energy that is dissipated and heating the solar photosphere. They proposed that when this magnetic heating is reduced due to lower magnetic activity the solar surface radiance could be reduced to close the lower end of the energy distribution (see Fig. 1 of Egorova et al., $2018 \mathrm{~b}$ ). This approach leads to a TSI decrease of $6 \mathrm{~W} / \mathrm{m}^{2}$ for the Maunder minimum (see Fig. 2). The authors have assumed that the reduced distribution is represented by the faint supergranule cell interior because this is the region on the Sun with the least amount of magnetic heating. As this assumption has a large uncertainty the authors give a factor of two for the uncertainty of their TSI reconstruction.

As discussed above, it is possible that direct solar forcing is amplified by up to a factor of two as only large amplification factors have been excluded by observations. This moderate amplification includes possible contributions from both mechanisms, Bottom-up as well as Top-down. With both terms in equation (2) contributing equally, a direct solar forcing of the Maunder minimum by $-10 \mathrm{~W} / \mathrm{m}^{2}$ amplified by a factor of two, one degree cooler climate during the 17 th century could be explained. With this reasoning, it is anticipated that it is the third statement, which is not correct.

Acknowledgments. I thank Y. Aono for providing data from his research in digital form, the editors of Nicollier et al. (2015), C. Nicollier and V. Gass, for permission to use part of chapter 3.2, Eugene Rozanov for providing unpublished results, and Stephan Nyeki for English language editing. The use of SORCE/TIM TSI data (vs. 18) from the NASA SORCE Mission Team and of TSIS/TIM TSI (vs. 3) data from the NASA TSIS Mission Team is acknowledged. The HadCRUT5 global annual mean temperature data are obtained from Climatic Research Unit (University of East Anglia) and Met Office. I thank Jean Lilensten for his valuable comments on a draft version.

\section{References}

Abbot CG. 1950. What causes weather. Leaflet of the Astronomical Society of the Pacific 6(254): 28-35.

Abreu JA, Beer J, Steinhilber F, Christl M, Kubik PW. 2013. ${ }^{10} \mathrm{Be}$ in ice cores and ${ }^{14} \mathrm{C}$ in tree rings: Separation of production and climate effects. Space Sci Rev 176(1-4): 343-349. https://doi.org/ 10.1007/s11214-011-9864-y. 
Anet JG, Muthers S, Rozanov EV, Raible CC, Stenke A, et al. 2014. Impact of solar versus volcanic activity variations on tropospheric temperatures and precipitation during the Dalton Minimum. Climate of the Past 10(3): 921-938. https://doi.org/ 10.5194/cp-10-921-2014.

Anet JG, Rozanov EV, Muthers S, Peter T, Brönnimann S, et al. 2013. Impact of a potential 21 st century "grand solar minimum" on surface temperatures and stratospheric ozone. Geophys Res Lett 40(16): 4420-4425. https://doi.org/10.1002/grl.50806.

Aono Y. 2015. Cherry blossom phenological data since the seventeenth century for Edo (Tokyo), Japan, and their application to estimation of March temperatures. Int J Biometeorology 59: 427-434. https://doi.org/10.1007/s00484-014-0854-0.

Aono Y, Kazui K. 2008. Phenological data series of cherry tree flowering in Kyoto, Japan, and its application to reconstruction of springtime temperatures since the 9 th century. Int J Climatology 28: 905-914. https://doi.org/10.1002/joc.1594.

Aono Y, Saito S. 2010. Clarifying springtime temperature reconstructions of the medieval period by gap-filling the cherry blossom phenological data series at Kyoto, Japan. Int J Biometeorology 54: 211-219. https://doi.org/10.1007/s00484-009-0272-x.

Arfeuille F, Rozanov E, Peter T, Fischer AM, Weisenstein D, Brönnimann S. 2010. Modeling the "Year without summer 1816" with the CCM SOCOL. In: EGU General Assembly Conference Abstracts, EGU General Assembly Conference Abstracts 12247.

Aschwanden MJ, Scholkmann F, Béthune W, Schmutz W, Abramenko V, et al. 2018. Order out of randomness: Self-organization processes in astrophysics. Space Sci Rev 214(2): 55. https://doi. org/10.1007/s11214018-0489-2.

BenMoussa A, Gissot S, Schühle U, Del Zanna G, Auchère F., et al. 2013. On-orbit degradation of solar instruments. Sol Phys 288(1): 389-434. https://doi.org/10.1007/s11207-013-0290-z.

Caballero-Lopez RA, Engelbrecht NE, Richardson JD. 2019. Correlation of long-term cosmicray modulation with solar activity parameters. Astrophys J 883(1): 73. https://doi.org/10.3847/1538$4357 / \mathrm{ab} 3 \mathrm{c} 57$.

Campisano CJ. 2012. Milankovitch cycles, paleoclimatic change, and hominin evolution. Nature Education Knowledge 4(3): 5.

Clette F, Vaquero JM, Cruz Gallego M, Lefèvre L. 2020. Sunspot and group number: Recent advances from historical data. In: $I A U$ General Assembly, pp. 156-159. https://doi.org/10.1017/ S174392131900396X.

Dewitte S, Crommelynck D, Mekaoui S, Joukoff A. 2004. Measurement and uncertainty of the longterm total solar irradiance trend. Sol Phys 224: 209-216. https://doi.org/10.1007/s11207005-5698-7.

Dudok de Wit T, Kopp G, Fröhlich C, Schöll M. 2017. Methodology to create a new total solar irradiance record: Making a composite out of multiple data records. Geophys Res Lett 44: 1196-1203. https://doi.org/10.1002/2016GL071866.

Dudok de Wit T, Ermolli I, Haberreiter M, Kambezidis H, Lam MM, et al. 2016. Earth's climate response to a changing Sun. EDP Sciences, France ISBN 978-2-7598-1733-7. https://doi.org/ 10.1051/978-2-75981733-7.

Eddy JA. 1976. The maunder minimum. Science 192(4245): 1189-1202. https://doi.org/10.1126/science.192.4245.1189.

Eddy JA, Gilliland RL, Hoyt DV. 1982. Changes in the solar constant and climatic effects. Nature 300(5894): 689-693. https://doi.org/10.1038/300689a0.

Egorova T, Rozanov E, Arsenovic P, Peter T, Schmutz W. 2018a. Contributions of natural and anthropogenic forcing agents to the early 20th century warming. Front Earth Sci 6: 206. https://doi. org/10.3389/feart.2018.00206.
Egorova T, Schmutz W, Rozanov E, Shapiro AI, Usoskin I, Beer J, Tagirov RV, Peter T. 2018b. Revised historical solar irradiance forcing. Astron Astrophys 615: A85. https://doi.org/10.1051/0004$6361 / 201731199$.

Egorova T, Schmutz W, Sukhodolov T, Rozanov E. 2019. Quantification of solar irradiance forcing by combining climate and phenological models. In: PMOD/WRC Annual Report 201823. https://www.pmodwrc.ch/en/institute/publications/annual-reports.

Fröhlich C. 2006. Solar irradiance variability since 1978. Revision of the PMOD composite during Solar Cycle 21. Space Sci Rev 125(1-4): 53-65. https://doi.org/10.1007/s11214-006-9046-5.

Fröhlich C. 2013. Total solar irradiance data. Space Sci Rev 176(1-4): 237-252. https://doi.org/10.1007/s11214-0119780-1.

Kapyla M, Solanki S, Usoskin I. 2016. Understanding solar activity. In: Earth's climate response to a changing Sun. Dudok de Wit T, Ermolli I, Haberreiter M, et al. (Eds.), pp. 97-102. ISBN 978-27598-1733-7. https://doi.org/10.1051/978-2-7598-1733-7.

Kawamura K, Parrenin F, Lisiecki L, Uemura R, Vimeux F, et al. 2007. Northern Hemisphere forcing of climatic cycles in Antarctica over the past 360,000 years. Nature 448(7156): 912-916. https://doi.org/10.1038/nature06015.

Kopp G, Lawrence G, Rottman G. 2005. The Total Irradiance Monitor (TIM): Science Results. Sol. Phys. 230(1-2): 129-139. https://doi.org/10.1007/s11207-005-7433-9.

Kopp G, Lean JL. 2011. A new, lower value of total solar irradiance: Evidence and climate significance. Geophys Res Lett 38(1): L01706. https://doi.org/10.1029/2010GL045777.

Lübken F-J, Nicoll K. 2016. Ground-based observations. In: Earth's climate response to a changing Sun. Dudok de Wit T, Ermolli I, Haberreiter M, et al., (Eds.) EDP Sciences, France, pp. 139-153. ISBN 978-27598-1733-7. https://doi.org/10.1051/978-2-75981733-7.

Marcott SA, Shakun JD, Clark PU, Mix AC. 2013. A reconstruction of regional and global temperature for the past 11,300 Years. Science 339(6124): 1198-1201. https://doi.org/10.1126/science. 1228026.

Matthes K, Haigh J, Hanslmeier A. 2016. The impact of solar variability on climate. In: Earth's climate response to a changing Sun. Dudok de Wit T, Ermolli I, Haberreiter M, et al. (Eds.) EDP Sciences, France, pp. 13-18. ISBN 978-2-7598-1733-7. https://doi.org/10.1051/978-2-7598-1733-7.

Maycock A, Misios S. 2016. "Top-down" versus "Bottom-up" mechanisms for solar-climate coupling. In: Earth's climate response to a changing Sun. Dudok de Wit T, Ermolli I, Haberreiter M, et al., (Eds.) EDP Sciences, France, pp. 237-246, ISBN 978-2-75981733-7. https://doi.org/10.1051/978-2-7598-1733-7.

Morice CP, Kennedy JJ, Rayner NA, Winn J, Hogan E, Killick R, Dunn R, Osborn T, Jones P, Simpson I. 2020. An updated assessment of near-surface temperature change from 1850: the HadCRUT5 dataset. $J$ Geophys Res Atmos 117(D8): e2019JD032352. https://doi.org/10.1029/2019JD032352.

Nicollier C, Bonnet R, Schildknecht T, Neuenschwander D, Schmutz W. 2015. Chapter 3.2 Weather and Climate. In: Our Space Environment, Opportunities, Stakes and Dangers. Nicollier C, Gass V, (Eds.) EFPL Press, Lausanne. ISBN 9781498751834.

Omoto Y, Aono Y. 1991. Effect of urban warming on blooming of Prunus yedoensis. Energy Build 15: 205-212. https://doi.org/ 10.1016/0378-7788(90)90132-3.

Owens MJ, Lockwood M, Hawkins E, Usoskin I, Jones GS, Barnard L, Schurer A, Fasullo J. 2017. The Maunder minimum and the Little Ice Age: an update from recent reconstructions and climate simulations. J Space Weather Space Clim 7: A33. https://doi.org/ $10.1051 / \mathrm{swsc} / 2017034$. 
Prša A, Harmanec P, Torres G, Mamajek E, Asplund M, et al. 2016. Nominal values for selected solar and planetary quantities: IAU 2015 Resolution B3. Astron J 152(2): 41. https://doi.org/10.3847/ $00046256 / 152 / 2 / 41$.

Rypdal K, Nilsen T. 2016. Observations on paleoclimatic time scales. In: Earth's climate response to a changing Sun. Dudok de Wit T, Ermolli I, Haberreiter M, et al., (Eds.) EDP Sciences, France, pp. 129-138. ISBN 978-2-7598-1733-7. https://doi.org/ 10.1051/978-2-7598-1733-7.

Schmutz W, Fehlmann A, Finsterle W, Kopp G, Thuillier G. 2013. Total solar irradiance measurements with PREMOS/PICARD. In: American Institute of Physics Conference Series, vol. 1531 of pp. 624-627. https://doi.org/10.1063/1.4804847.

Schmutz W, Haberreiter M. 2016. Orbital forcing of glacial interglacial cycles. In: Earth's climate response to a changing Sun. Dudok de Wit T, Ermolli I, Haberreiter M, et al., (Eds.) EDP Sciences, France, pp. 103-109, ISBN 978-2-7598-1733-7. https://doi.org/10.1051/978-2-7598-1733-7.

Shapiro AI, Schmutz W, Rozanov E, Schoell M, Haberreiter M, Shapiro AV, Nyeki S. 2011. A new approach to the long-term reconstruction of the solar irradiance leads to large historical solar forcing. Astron Astrophys 529: A67. https://doi.org/10.1051/00046361/201016173.

Shapiro AI, Solanki SK, Krivova NA, Cameron RH, Yeo KL, Schmutz WK. 2017. The nature of solar brightness variations. Nature Astron 1: 612-616. https://doi.org/10.1038/s41550-017-0217-y.

Solanki SK, Krivova NA, Haigh JD. 2013. Solar Irradiance Variability and Climate. Ann Rev Astron Astrophys 51(1): 311-351. https://doi.org/10.1146/annurev-astro-082812-141007.

Sukhodolov T, Egorova T, Stenke A, Ball WT, Brodowsky C, Chiodo G, Feinberg A, Friedel M, Karagodin-Doyennel A, Peter T, Vattioni S, Rozanov E. 2021. Atmosphere-ocean-aerosol-chemistryclimate model SOCOLv4.0: description and validation. Geosci Model Dev Discuss. [preprint], https://doi.org/10.5194/gmd-2021-35, in review.

Sukhodolov T, Rozanov E, Shapiro AI, Anet J, Cagnazzo C, Peter T, Schmutz W. 2014. Evaluation of the ECHAM family radiation codes performance in the representation of the solar signal. Geosci Model Dev 7(6): 2859-2866. https://doi.org/ 10.5194/gmd-7-2859-2014.

Urban SE, Seidelmann PK. 2012. Explanatory supplement to the astronomical almanac, 3rd edn. University Science Books, US, Sausalito, United States.

Usoskin IG, Hulot G, Gallet Y, Roth R, Licht A, Joos F, Kovaltsov GA, Thebault E, Khokhlov A. 2014. Evidence for distinct modes of solar activity. Astron Astrophys 562: L10. https://doi.org/ 10.1051/0004-6361/201423391.

van Loon H, Meehl GA, Shea DJ. 2007. Coupled air-sea response to solar forcing in the Pacific region during northern winter. J Geophys Res Atmos 112(D2): D02108. https://doi.org/10.1029/ 2006JD007378.

Wild M, Folini D, Hakuba M, Schär C, Seneviratne S, Kato S, Rutan D, Ammann C, Wood E, König-Langlo G. 2015. The energy balance over land and oceans: An assessment based on direct observations and CMIP5 climate models. Clim Dyn 44: 3393-3429. https://doi. org/10.1007/s00382-014-2430-z.

Willson RC, Mordvinov AV. 2003. Secular total solar irradiance trend during solar cycles 21-23. Geophys Res Lett 30(5): 1199. https://doi.org/10.1029/2002GL016038.

Yeo KL, Solanki SK, Krivova NA, Rempel M, Anusha LS, Shapiro AI, Tagirov RV, Witzke V. 2020. The dimmest state of the Sun. Geophys Res Lett 47(19). https://doi.org/1029/2020GL090243.

\section{Appendix}

\section{Regression analyses of reconstructed March temperatures in Japan}

Inspecting Figure 1 it can be seen that the March temperatures in Kyoto and Edo bay have different trends with time. One important information to clarify is whether this is an artifact of the transformation from blossom date to temperature or whether there are different temperature trends at the two locations.

Individual annual dates of cherry blossom flowering dates have been collected from historic archives by Aono \& Kazui (2008) and Aono (2015). In the left panel of Figure A.1 annual flowering dates as well as 15-year averages are shown and it can be seen that also the flowering dates at the two locations have different trends. The flowering dates for Edo bay are later than the flowering dates for Kyoto in the 17 th century. The relation is inversed in the 19th century when the average flowering dates for Edo bay are earlier than for Kyoto. Thus, there is a genuine systematic trend between average March temperatures at the two locations.

Aono (2015) has provided temperatures that are smoothed by a 31-year linear regression. The energy dose model by Aono \& Kazui (2008) and Aono (2015) yields a linear relation between the flowering date and temperature but the relations for the two locations are slightly different: For Edo bay the relation is $-3.94 \mathrm{DoY} /{ }^{\circ} \mathrm{C}$ and for Kyoto the DoY-temperature relation is $-3.53 \mathrm{DoY} /{ }^{\circ} \mathrm{C}$. This allows to build 15 -year averages of March temperatures at both locations. In the right panel of Figure A.1 the temperature differences at the two locations are illustrated as a function of time. Year-to-year variations of the blossom dates have a standard deviation of $1.25{ }^{\circ} \mathrm{C}$ and using a $15-\mathrm{yr}$ average yield a reduced $1 \sigma$ uncertainty of the order of $\pm 0.3{ }^{\circ} \mathrm{C}$ if all years within a 15 -yr interval have a historic flowering record. Accordingly, the differences should have an uncertainty of $0.4{ }^{\circ} \mathrm{C}$. This is confirmed by the deviations from a linear regression that has a standard variation $0.38{ }^{\circ} \mathrm{C}$. These sigma values imply that the mean temperature of the two locations have random uncertainties of $\sigma_{\text {mean }} \approx 0.2{ }^{\circ} \mathrm{C}$ and the 15-year averages at one location have uncertainties of $0.3{ }^{\circ} \mathrm{C}$. The significant linear dependence $(P=0.01 \%)$ of the temperature difference with time indicates that there is a systematic difference between the March temperatures at the two locations, whatever the reason, e.g., evolution of a species, a change of the cherry tree species, or a specific climatic difference between the two locations.

The relevant information derived above for the investigation of the role of solar forcing is that there is a systematic bias of the reconstructed temperatures with time. However, as shown in Table A.1, for the epoch interval 1650-1890 the resulting correlation between temperatures and solar forcing is robust and does not depend strongly on this bias. The March temperatures at both locations are strongly correlated (with significance) to TSI, no matter what combination is used: Individual locations or the mean of both locations or if a multiple regression with dependencies on time and TSI is calculated, or only TSI is used as an independent parameter. The correlation to solar forcing turns out to be as strong as the correlations between the temperature variations at the two locations (first two rows of Tab. A.1). 

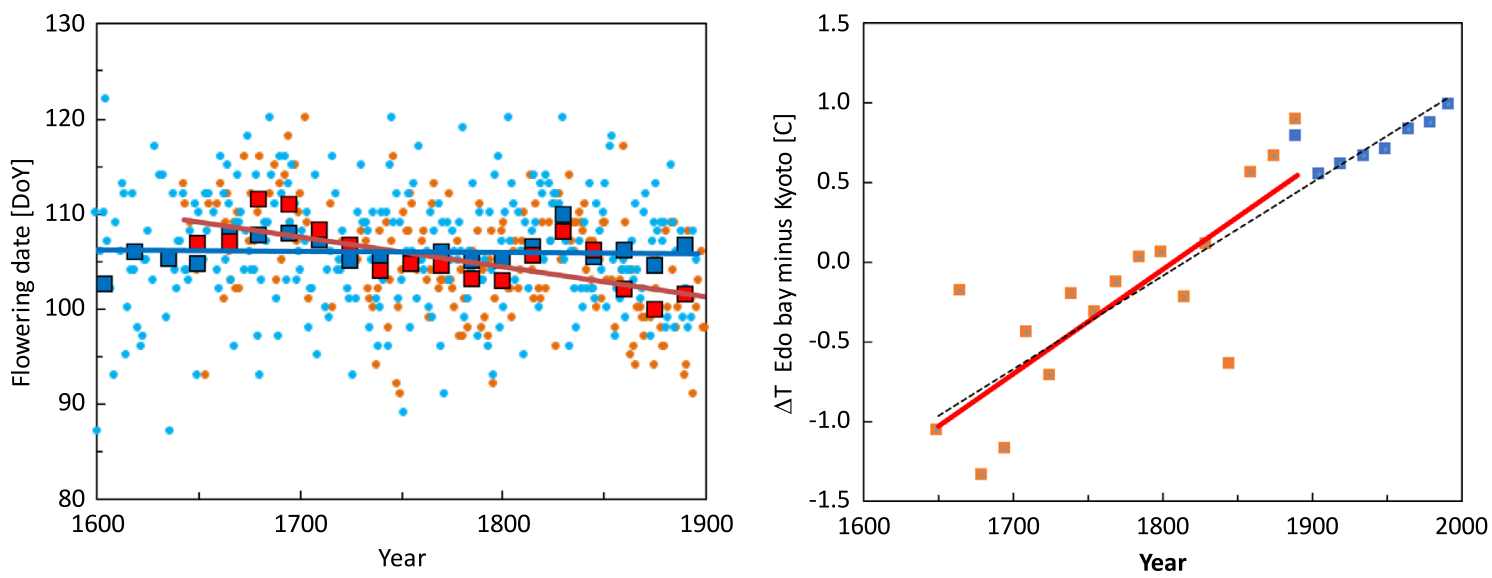

Fig. A.1. Left panel: Annual cherry blossom flowering dates given as a day of the year (DoY) in Kyoto (blue dots) and Edo bay (red dots) (Aono \& Kazui, 2008; Aono, 2015). The blue and red squares denote 15-year averages for Kyoto and Edo bay, respectively, and the lines indicate linear trends of the flowering dates. Right panel: The red squares indicate differences of 15-year averaged reconstructed March temperatures between Kyoto and Edo bay as a function of the time (red squares). The blue symbols are differences of thermometer measured temperatures smoothed by a 31-year linear regression from 1890 to 1990.

Table A.1. Linear Regression correlation coefficients, $R, c_{\mathrm{TSI}}, c_{\text {time }}$, and probability of zero hypothesis, $P_{\mathrm{TSI}}$. The number of independent points is given by a 15-year interval and the fitted time interval (except for the last row including years before 1400, for which several 15-year averages with less than 10 historic records have been omitted). The values of column $\sigma$ are the standard deviations of the differences to the predicted relation.

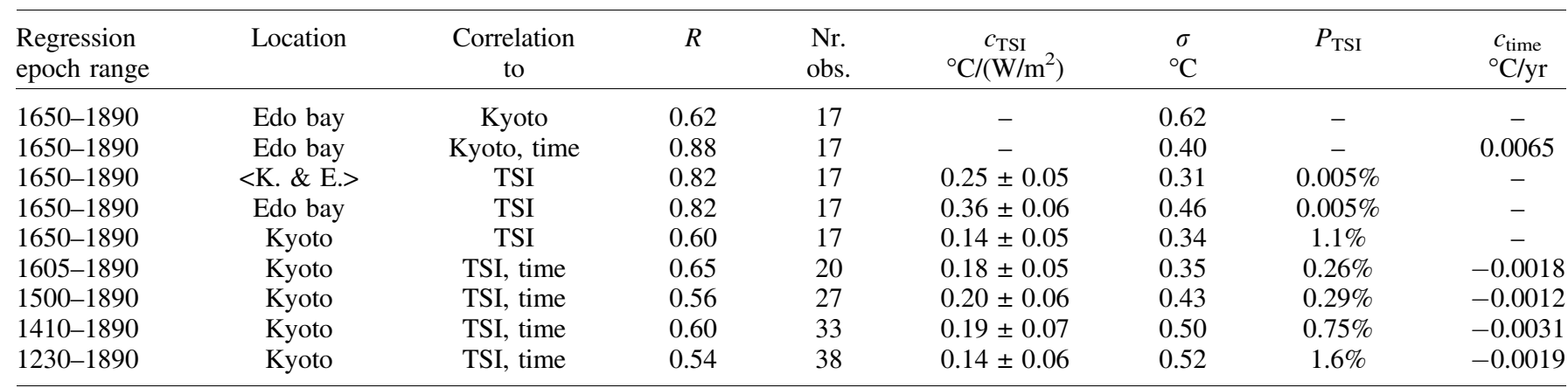

In the left panel of Figure A.2, the dependence of the mean of the temperatures at Kyoto and Edo bay on TSI is illustrated. In the right panel of Figure A.2, the residuals are plotted as a function of time after subtracting the linear relation of temperature versus TSI. It can be seen that a systematic influence possibly starts as early as mid 19th century but at the latest around the year 1900. The regression is strong $(R=0.82)$ and the probability that the relation is by chance is $P=0.005 \%$. Thus, the probability that solar forcing has influenced the temperature variations at Kyoto and Edo bay from 1650 to 1890 is higher than $99.99 \%$.

In Table A.1, the regression coefficients are listed for several time intervals. As can be seen, the probability of zero hypothesis is always below the significance level of $5 \%$ and thus, solar forcing is significant in mid-southern Japan not only in the
Maunder minimum in the 17 th century but over the whole time interval of almost 700 years. The only caveat is that there is a need to allow for a linear time bias if longer time intervals are considered. This is illustrated in Figure A.3 showing the comparison between solar forcing and reconstructed temperature at Kyoto with and without bias correction of the reconstructed Kyoto temperature.

The residuals of the multiple linear regression with the independent variables TSI and time are shown in Figure A.4. The linear regression to the residuals has a zero slope because the bias has been fitted with time as an independent parameter. However, it can be seen that the residuals are not randomly distributed. A higher-order fit with time would reduce some of the remaining bias. 

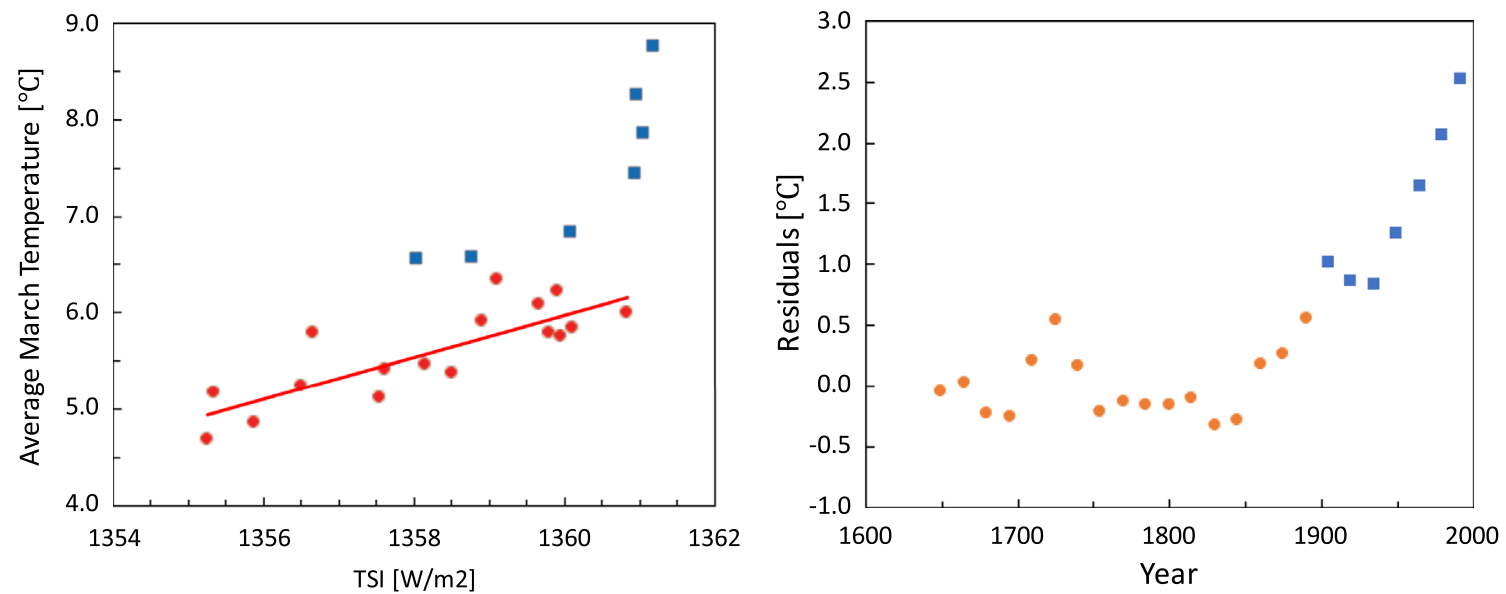

Fig. A.2. Left panel: 15-year averages of the mean reconstructed March temperatures of Kyoto and Edo bay (red dots) within the years 1650 1890 as a function of 15-year averages of TSI. The blue squares denote 15-year averages of thermometer measured temperatures in the time interval 1890-1995. Right panel: Residuals from the linear regression of the left panel (third row of Tab. A.1) as a function of time.

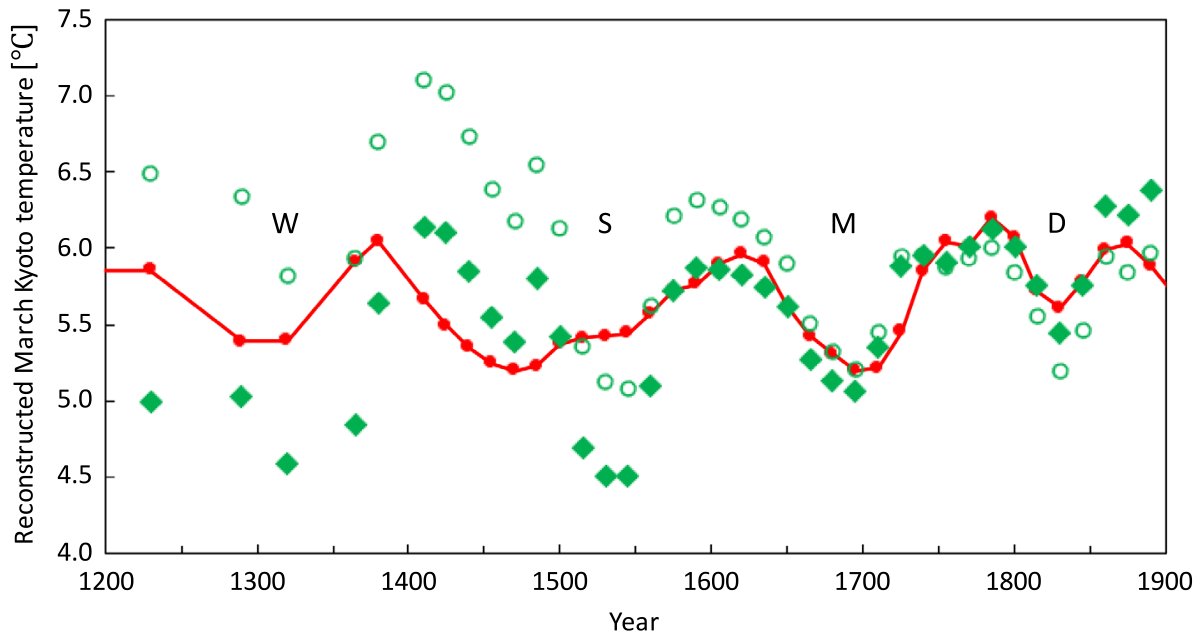

Fig. A.3. Reconstructed averaged March temperatures at Kyoto, Japan (open green circles) adapted from Aono \& Kazui (2008) and bias-corrected temperatures (green diamonds), compared to temperatures calculated from 15-year averages of TSI according to Egorova et al. (2018b) (red line). The reconstructed temperatures are 15-year averages for which at least 10 yearly values are available. For years later than 1400 , there are no gaps of 15-year averaged temperature records but for earlier years there are gaps in the 15-year sampled record. The coefficients $c_{\text {TSI }}$ to calculate a temperature from a TSI value and $c_{\text {time }}$ to calculate the linear time-bias are given in Table A.1 in the row $1410-$ 1890. Grand solar activity minima periods are indicated: W Wolf, S Spörer, M Maunder, and D Dalton minimum. 
W.K. Schmutz: J. Space Weather Space Clim. 2021, 11, 40

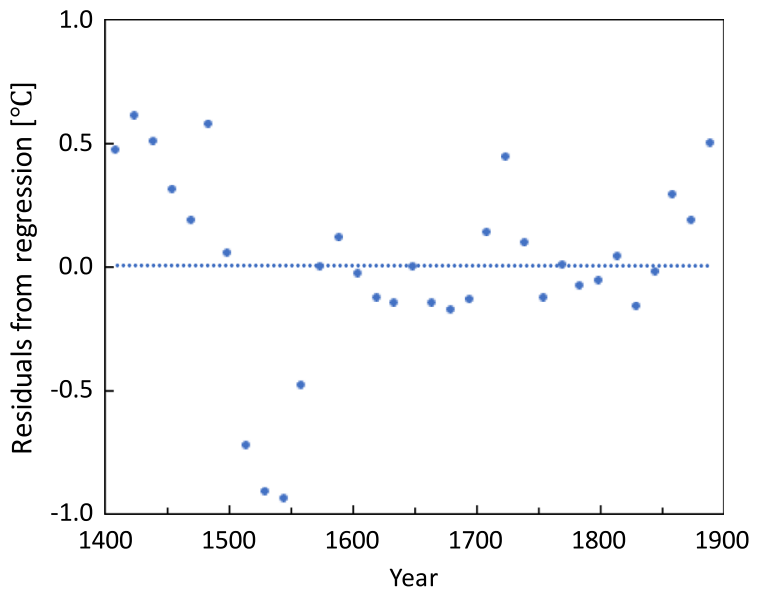

Fig. A.4. Residuals from the multi-linear regression between reconstructed March temperatures at Kyoto and solar forcing and a linear bias with time as shown in Figure A.3.

Cite this article as: Schmutz W.K. 2021. Changes in the Total Solar Irradiance and climatic effects. J. Space Weather Space Clim. 11, 40. https://doi.org/10.1051/swsc/2021016. 